\title{
Accuracy analysis of the RSSI BLE SensorTag signal for indoor localization purposes
}

\author{
Mariusz Kaczmarek \\ Gdansk University of Technology Narutowicza 11/12, 80- \\ 233 Gdansk, Poland \\ Email: mariusz.kaczmarek@eti.pg.gda.pl
}

\author{
Jacek Ruminski, Adam Bujnowski \\ Gdansk University of Technology Narutowicza \\ 11/12, 80-233 Gdansk, Poland \\ Email: \{jwr, bujnows\}@biomed.eti.pg.gda.pl\}
}

\begin{abstract}
In this paper we describe possibility of use the RSSI signal (Radio Signal Strength Indication) from Texas Instruments SensorTag $\mathrm{CC2650}$ for indoor positioning purposes. This idea is not a new but in our opinion it is possible to use SensorTags with Bluetooth LE wireless interface for positioning inside buildings in such applications as people findings in hospitals, senior come care, etc. RSSI is mostly selected as the sensor localization method in the indoor circumstances. In this paper, we aim to analyze accuracy, calibrate and map RSSI to distance by doing a series of the experiments. Obtained results are very promising and shows possibility of use this technique for position estimation.
\end{abstract}

\section{INTRODUCTION}

DEA of position localization basing on wireless networks is widely known for mobile phones where using information about signal strength from BTS (Base Transceiver Station) one can determine the position of mobile phone speaker [1]. However, this is a coarse location, which is not suitable for indoors use.

Many technologies have been investigated to bridge the gap and bring positioning indoors, such as a combination of AGPS, accelerometer and magnetometer [2], Bluetooth [3], Ultrawideband [4], ZigBee [5]. Wi-Fi is one of most discussed of them, and is considered as the most promising one as the infrastructure and user equipment is already widely available, e.g. in public buildings, public area like parks, airports or railway stations, and it is able to deliver accuracies in the range of a few meters. An exemplary distribution of wireless networks in the building of the Faculty of Electronics, Telecommunications and Informatics of Gdansk University of Technology is shown in Fig. 1. One can choose the best configuration for signal strength scanning. Wi-Fi fingerprinting was pioneered in [6], and has

This work was supported by ERA-NET-CHIST-ERA II eGLASSES - The interactive eyeglasses for mobile, perceptual computing; and by European Regional Development Fund concerning the project UDA-POIG.01.03.01-22-139/09-03 -"Home assistance for elders and disabled - DOMESTIC", Innovative Economy 2007-2013, National Cohesion Strategy since attracted considerable interest, mainly focused on increasing the accuracy of the technique.

But the use of Wi-Fi network access points can be limited due to different artifacts such as different sensitivity of chipsets in mobile devices [7][8]. Some efforts have also been made in the literature to reduce the effects of RSS variations due to channel impediments by using a compressive sensing (CS) principle such as in [9].

For private home position localization more suitable will be use of bluetooth devices called beacons or like e.g. Texsas Instruments BLE SensorTag CC2650 [10]. This is cheap devices for controlling environmental parameters like ambient temperature, humidity, air pressure, luxometer data and accelerometer data. A good example of implementation of the indoor positioning system is Nashvile project: Mayor, Music City Center Unveil Wayfinding App [11].

\section{MATERIAL AND METHODS}

\section{A. Bluetooth LE}

The Bluetooth 1.0 standard was introduced by SIG in 1999 [12]. The new specification of Bluetooth 4.0LE improved technology that helps everyday gadgets stay paired longer while using less power. Bluetooth 4.0 enables a new class of gadgets such as fitness trackers, medical devices, key fobs for car, beacons sensors and even home lighting controls.

\section{B. Bluetooth and Wi-Fi possible interference}

Because both Wi-Fi and Bluetooth wireless technology share $2.4 \mathrm{GHz}$ frequency and spectrum and will often be located in close physical proximity to one another, there is concern for how they may interfere with one another. Fig. 1. shows the Wi-Fi networks and spectrum at Faculty of Electronics, Telecommunication and Informatics GUT. Wi$\mathrm{Fi}$ and Bluetooth fail gracefully in the presence of interference. By this is meant that the communication protocols are very robust and include mechanisms for error checking and correcting, as well as requesting that corrupted packets be resent. Therefore the result of increasing levels of 
interference is almost always confined to a slowing of the data rate as more packets need to be resent.

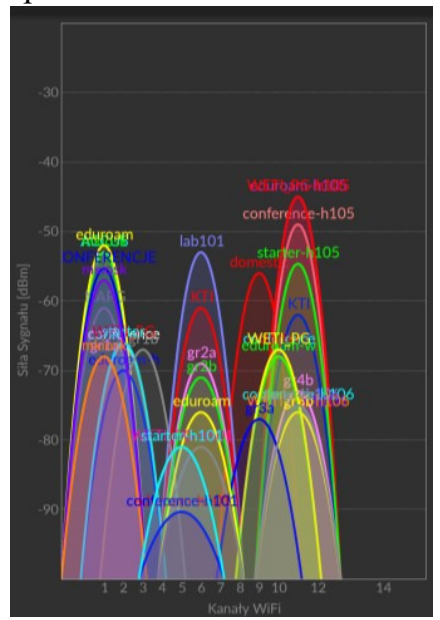

Fig. 1 Wi-Fi network spectrum measurements

\section{RSSI measurements and distance calculations}

Received signal strength indication (RSSI) is a measurement of the power level received by sensor. Because radio waves propagate according to the inverse-square law, distance can be approximated based on the relationship between transmitted and received signal strength (the transmission strength is a constant based on the equipment being used), as long as no other errors contribute to faulty results. Various experts have given nice theoretical ideas of the model propagation. These theoretical ideas prepares ground for starting and estimating the parameters.

$$
R S S I=-10 \mathrm{n} \cdot \log (d)+A,
$$

where $\mathrm{d}=$ distance, $\mathrm{A}=$ txPower at distance of $1 \mathrm{~m}, \mathrm{n}=$ signal propagation constant, usually in free space $n=2$, but it will vary based on local geometry - for example, a wall will reduce RSSI by $\sim 3 \mathrm{dBm}$ and will affect $n$ accordingly and $[\mathrm{RSSI}]=\mathrm{dBm}$.

However, practically you need to measure RSSI value at different known and convenient distances and prepare chart. This measurements should be done in various seasons (day, night, dry, cold, rainy). These charts will provide you procedure of extrapolation or intrapolation of the distance.

\section{Position estimations}

Based on the coordinates of three reference nodes: $\mathrm{ST} \# 1(\mathrm{x} 1, \mathrm{y} 1), \mathrm{ST} \# 2(\mathrm{x} 2, \mathrm{y} 2)$, and ST\#3(x3, y3), and the corresponding distances (calculated from RSSI measured signal) from each reference node to the target node: R1, R2, and R3, we can obtain the following equations [13]:

$$
\begin{aligned}
& \left(x_{1}-x\right)^{2}+\left(y_{1}-y\right)^{2}=R_{1}^{2} \\
& \left(x_{2}-x\right)^{2}+\left(y_{2}-y\right)^{2}=R_{2}^{2}, \\
& \left(x_{3}-x\right)^{2}+\left(y_{3}-y\right)^{2}=R_{3}^{2}
\end{aligned}
$$

where (x, y) denotes the (unknown) coordinates of the target P.

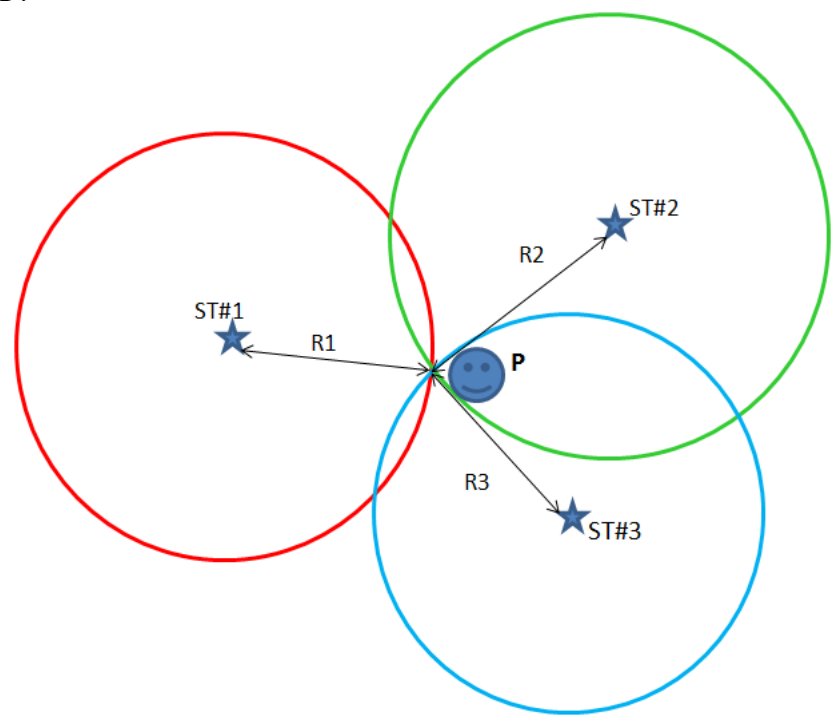

Fig. 2 Position estimation basing on three distances from SensorTags ST\#1. ST\#2, ST\#3

\section{EXPERIMENTS AND RESULTS}

We perform several experiments in different configurations. Two of them are shown in Fig. 3. Firs case is called direct view because SensorTags array was in direct view with receiving smartphone without any obstacles, the second one was called with wall obstacle because the sensor array was hidden behind the wall's corner.
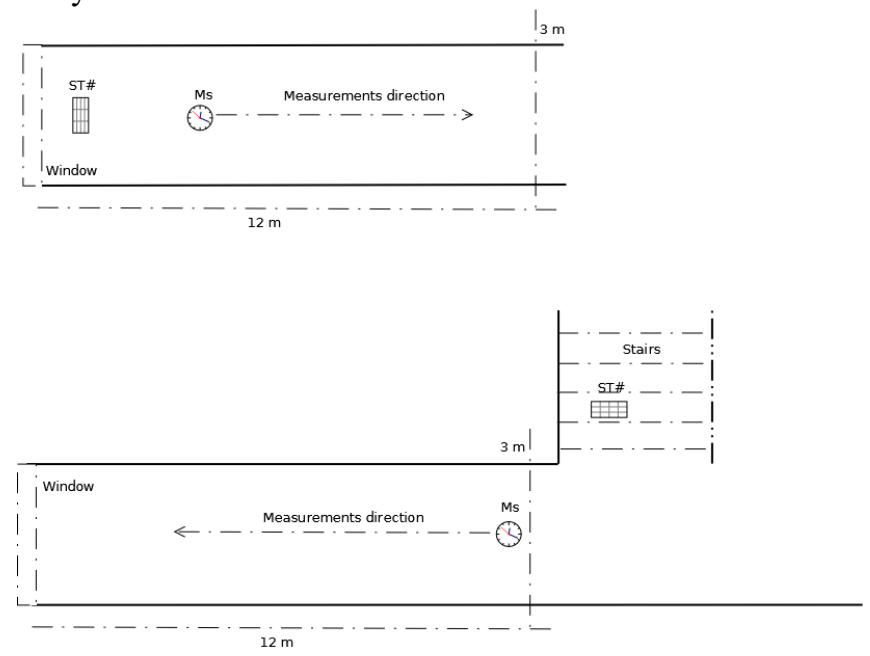

Fig. 3 Configuration of measurements procedure of RSSI (CC2650); a) direct view, b) with wall obstacle

Measurements was done using the written application (Fig. 8) - equipment: smartphone: One Plus One, Android 5.1.1, API 22 TI Sensor Tag CC2650. For fixed distance (range: $0.1 \mathrm{~m}$ up to $6 \mathrm{~m}$ ) it was read RSSI value for the three devices that radiate towards the receiver ( $\mathrm{n}=10$ readings for each device). Results for both configuration are shown in Fig. 4 and Fig. 5. 


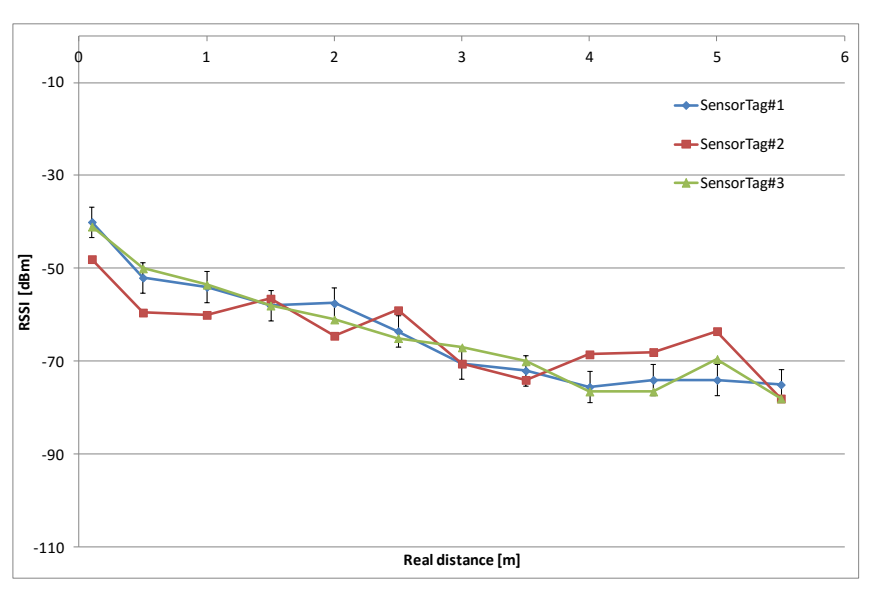

Fig. 4 RSSI vs distance measurements for three TI CC2650 SensorTags - direct view

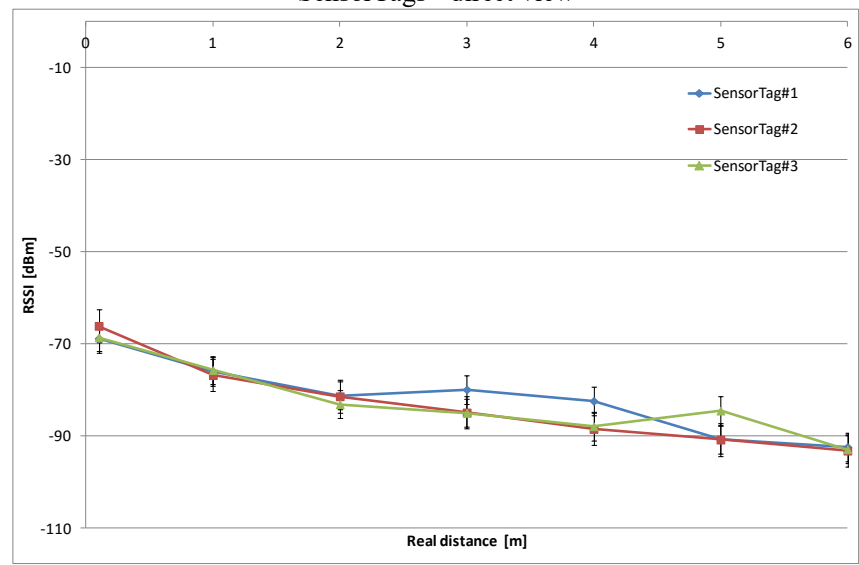

Fig. 5 RSSI vs distance measurements for three TI CC2650 SensorTags with wall obstacle

Basing on obtained readings the mean values and standard deviation values were calculated and plotted (RSSI vs "known" distance) on the charts - Table I. Next the data were fitted to polynomial model according to equation (3).

$$
R S S I=\mathrm{A} \cdot d^{2}+B \cdot d+C,
$$

where: A, B, C - parameters, $d$ - distance.

It seems to be the best way to calibrate the system according to flat/home configurations such as walls, furniture and other obstacles. Example of fitted curve for measured data for SensorTag\#3 in direct view experiment is shown in Fig.6.

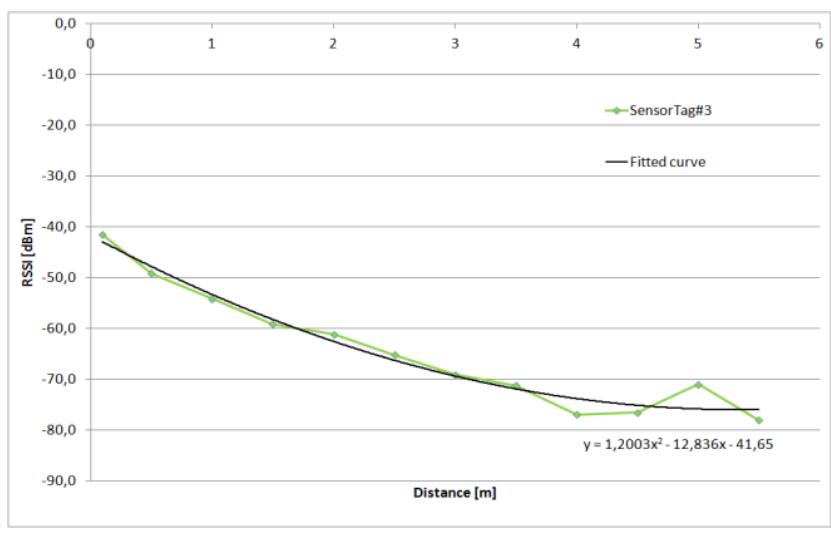

Fig. 6 Dependency between distance and RSSI. The continuous line represents the linear regression model. Dots are mean value of 10 measurements for each distance

\section{APPLICATION}

Possible scenarios for the use of the proposed method include: a hospitals, nursing homes, senior homes but also museums, airports or train stations.

One example [14] is shown in Fig. 7. and Fig. 8. Inside the senior's flat one can place TI CC2650 sensors for remote monitoring of the environmental conditions (ambient temperature, humidity, luminance). Added value of such Bluetooth LE (BLE) system is possibility of indoor target localization. Dedicated application (Fig. 8) with possibility of import flat schema and localization of sensor allows for calibration measurements of RSSI signal. Basing on this procedure it is possible to track target among short distances.

As there is no fixed standard which manufacturers are required to follow, signal strength indications are to be used for indication only and do not indicate the true absolute signal strength received. These values are reported by a piece of software which allows the operating system to use the wireless card - i.e. the drivers. These drivers feature the role of controlling and reporting the status of the card, and

TABLE I.

EXPERIMENTS RESULTS FOR DIRECT VIEW PROCEDURE, ONEPLUS ONE SMARTPHONE WITH ANDROID 6.0.1+ TI CC2650

\begin{tabular}{|c|c|c|c|c|c|c|c|c|c|c|c|c|c|c|c|}
\hline \multirow{2}{*}{$\begin{array}{r}\begin{array}{c}\text { Distance } \\
\text { [m] }\end{array} \\
0.1 \\
\end{array}$} & \multicolumn{3}{|c|}{$\begin{array}{l}\text { RSSI mean value } \\
{[\text { [dBm] }}\end{array}$} & \multicolumn{3}{|c|}{$\begin{array}{l}\text { RSSI median value } \\
{[\text { [dBm] }}\end{array}$} & \multicolumn{3}{|c|}{$\begin{array}{c}\text { Standard } \\
\text { deviation } \\
\text { [dBm] }\end{array}$} & \multicolumn{3}{|c|}{$\begin{array}{l}\text { Estimated distance - } \\
\text { equation (1) }[\mathrm{m}]\end{array}$} & \multicolumn{3}{|c|}{$\begin{array}{l}\text { Estimated distance - } \\
\text { calibrated model, } \\
\text { equation }(3)[\mathrm{m}]\end{array}$} \\
\hline & -42.0 & -40 & -48 & -41 & -48.0 & -41.5 & 4.7 & 3.5 & 1.8 & 0.08 & 0.12 & 0.07 & -0.02 & -0.60 & -0.01 \\
\hline 0.5 & -51.5 & -52 & -59.5 & -50 & -60.0 & -49.1 & 2.2 & 5.8 & 2.1 & 0.60 & 1.12 & 0.38 & 0.80 & 1.48 & 0.62 \\
\hline 1 & -54.2 & -54 & -60 & -53.5 & -59.3 & -54.1 & 3.3 & 4.7 & 1.7 & 1.00 & 1.00 & 1.00 & 1.06 & 1.34 & 1.08 \\
\hline 1.5 & -57.7 & -58 & -56.5 & -58 & -58.0 & -59.1 & 4.5 & 6.9 & 4.8 & 1.57 & 0.87 & 1.89 & 1.42 & 1.08 & 1.60 \\
\hline 2 & -58.7 & -57.5 & -64.5 & -61 & -64.2 & -61.1 & 2.6 & 3.7 & 1.4 & 1.78 & 1.77 & 2.41 & 1.53 & 2.42 & 1.83 \\
\hline 2.5 & -63.0 & -63.5 & -59 & -65 & -58.8 & -65.2 & 1.7 & 1.9 & 3.3 & 2.98 & 0.95 & 3.90 & 2.05 & 1.24 & 2.35 \\
\hline 3 & -73.0 & -70.5 & -70.5 & -67 & -69.1 & -69.1 & 6.9 & 5.0 & 5.9 & 9.05 & 3.03 & 6.04 & 3.84 & 3.85 & 2.95 \\
\hline 3.5 & -73.9 & -72 & -74 & -70 & -72.5 & -71.2 & 8.7 & 5.4 & 4.3 & 9.93 & 4.35 & 7.59 & 4.13 & 5.48 & 3.35 \\
\hline 4 & -75.9 & -75.5 & -68.5 & -76.5 & -68.0 & -76.9 & 6.2 & 2.5 & 7.3 & 12.18 & 2.70 & 13.65 & 6.23 & 3.48 & 6.89 \\
\hline 4.5 & -73.0 & -74 & -68 & -76.5 & -70.4 & -76.5 & 4.2 & 6.5 & 6.2 & 9.05 & 3.49 & 13.12 & 3.84 & 4.36 & 6.89 \\
\hline 5 & -73.6 & -74 & -63.5 & -69.5 & -65.4 & -71.0 & 5.7 & 4.5 & 4.0 & 9.63 & 2.03 & 7.43 & 4.03 & 2.73 & 3.31 \\
\hline 5.5 & -76.0 & -75 & -78 & -78 & -77.2 & -78.0 & 5.2 & 3.6 & 4.8 & 12.30 & 6.99 & 15.22 & 6.20 & 6.99 & 6.99 \\
\hline
\end{tabular}


therefore the strengths reported by the card are highly dependent on the mapping which is established between hardware analog to digital converse values and RSSI values reported by the driver.

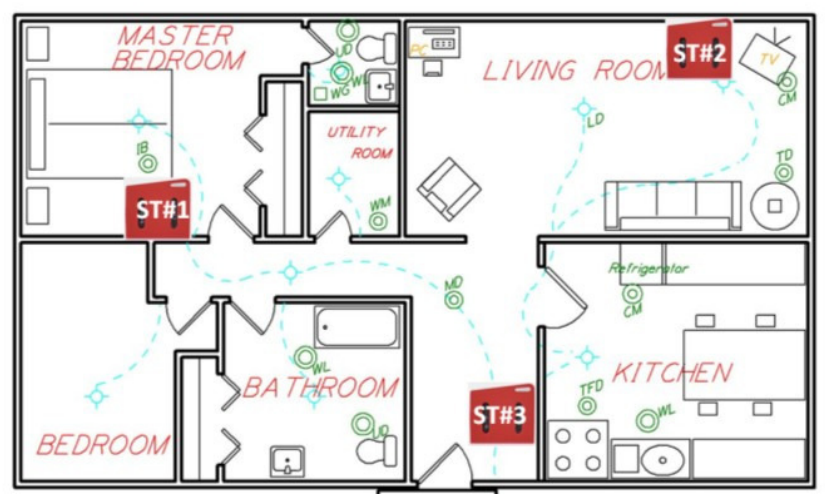

Fig. 7 Example of possible implementation in home environment for three TI CC2650 SensorTags

Different device design and usage by end users could also lead to different signal levels due to human influences. Furthermore, differences in the environment from interfering access points and devices, as well as human traffic and changes in furniture layout will cause different RSSIs to be received in the same location.

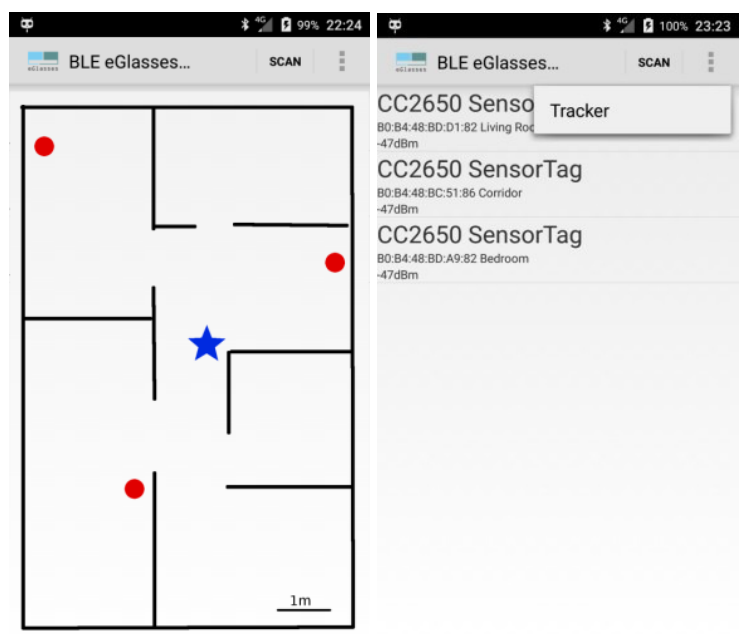

Fig. 8 GUI of mobile application for BLE indoor positioning

Disadvantages of the method:

- acceptable minimum version of Android is 4.2 (API 19)

- CC2560 - lack of continuous operation mode,

- very unstable RSSI readings.

\section{CONCLUSION}

It was argued that there are many factors which can affect the RSSI returned by a BLE devices, including the antenna design, hardware design, drivers and the environment. Given the large number of factors governing the received RSSI, calibration is unlikely to be able to compensate for all of them, leading us to conclude that there is an inherent limit to the accuracy of a BLE positioning system especially when multiple devices are used.

Small scale signal variations (e.g. multipath) may greatly affect the RSS measurement. Variations of up to $30-40 \mathrm{~dB}$ have been reported [15]. We have measured at least 2-6dB variations in indoor deployments of low power Bluetooth LE networks. Therefore, we would suggest that instead of using a single RSSI measurement to estimate distance, try using the average or median value of $\mathrm{N}$ measurements collected on the same spot (at least $\mathrm{N}>20$ ) so that you can reduce the effect of small scale fading. Then you can use the logdistance model with more accuracy. If you have more measurements, extract the basic characteristics of the propagation environment first (like path loss exponent etc), to achieve better results. Another interesting issue is the question of the deployment of BLE tag's in the home environment for optimal readings and determination of the position.

\section{REFERENCES}

[1] M. Sauter (2010). "3.7.1 Mobility Management in the Cell-DCH State". From GSM to LTE: An Introduction to Mobile Networks and Mobile Broadband. John Wiley \& Sons. ISBN 9780470978221.

[2] D. Gusenbauer, C. Isert, and J. Krosche, "Self-contained indoor positioning on off-the-shelf mobile devices", Indoor Positioning and Indoor Navigation 2010, pp. 1-9.

[3] S. Feldmann, K. Kyamakya, A. Zapater, and Z. Lue, "An indoor Bluetooth-based positioning system: concept, implementation and experimental evaluation", International Conference on Wireless Networks, 2003.

[4] C. Zhang, M. Kuhn, B. Merkl, A.E. Fathy, and M. Mahfouz, "Accurate UWB indoor localization system utilizing time difference of arrival approach", IEEE Radio and Wireless Symposium, pp. 515 518, 17-19th Oct. 2006.

[5] M. Sugano, "Indoor localization system using rssi measurement of wireless sensor network on zigbee standard", Wireless and Optical Communications, pp. 1-6, 2006.

[6] P. Bahl, V.N. Padmanabhan, "RADAR: an in-building RF-based user location and tracking system”, Proc. of Infocom, pp. 775-784, 2000.

[7] A. Parameswaran, H. Thottam, S. Upadhyaya, "Is RSSI a Reliable Parameter in Sensor Localization Algorithms - An Experimental Study" (PDF). September 2009. 28th International Symposium On Reliable Distributed Systems, New York. Retrieved 17 March 2013..

[8] L., Gough; Gallagher, Thomas; Binghao, Li. "Differences in RSSI readings made by different Wi-Fi chipsets: A limitation of WLAN localization". Localization and GNSS (ICL-GNSS), 2011 International Conference on.

[9] C. Feng, W. Au, S. Valaee, and Z. Tan, "Compressive sensing based positioning using rss of wlan access points," in INFOCOM, 2010 Proceedings IEEE, 2010, pp. 1-9.

[10] http://www.ti.com/sitesearch/docs/universalsearch.tsp?searchTerm=cc 2650\&linkId $=10 \&$ src $=$ top $\& \mathrm{~m}=\mathrm{dd} \#$ linkId $=3$

[11] http://www.nashville.gov/News-Media/News-Article/ID/3477/MayorMusic-City-Center-Unveil-Wayfinding-App.

[12] http://standards.ieee.org/about/get/802/802.15.html.

[13] G. Han, D. Choi, W. Lim, "A Novel Reference Node Selection Algorithm Based on Trilateration for Indoor Sensor Networks", IEEE Intl Conf. on Computer and Inform. Technology, 2007,p.1003-1008.

[14] M. Kaczmarek, A. Bujnowski, J. Wtorek, A. Poliński: Multimodal Platform for Continuous Monitoring of the Elderly and Disabled// Journal of Medical Imaging and Health Informatics.. -Vol. 2., issue 1 (2012), p.56-63.

[15] C. Feng, W. Au, S. Valaee, and Z. Tan, "Compressive sensing based positioning using rss of wlan access points," in INFOCOM, 2010 Proceedings IEEE, 2010, p. 1-9. 